\title{
Searching Building Blocks with Large Excited-State Gaps in Five-memebered Heterocyclic Rings by Aromaticity Strategy
}

Qi Sun ${ }^{1}$, Yuanyuan Cui ${ }^{3}$, Yishi Wu ${ }^{4}$, Can Gao ${ }^{5}$, Qi Ou ${ }^{1}$, Deping $\mathrm{Hu}^{1}$, Lu Wang $^{1}$, Yue Wang ${ }^{3}$, Huanli Dong $^{5}$, Qian Peng ${ }^{2,4 *}$, and Zhigang Shuai ${ }^{1 *}$

\author{
${ }^{1}$ MOE Key Laboratory of Organic Optoelectronics and Molecular Engineering \\ Department of Chemistry, Tsinghua University \\ Beijing, 100084, P. R. China \\ E-mail: zgshuai@tsinghua.edu.cn.
}

${ }^{2}$ School of Chemical Sciences

University of Chinese Academy of Sciences

Beijing, 100049, P. R. China

E-mail: qpeng@iccas.ac.cn, qianpeng@ucas.ac.cn

\author{
${ }^{3}$ State Key Laboratory of Supramolecular Structure and Materials \\ College of Chemistry, Jilin University \\ Changchun 130012, P. R. China
}

${ }^{4}$ Beijing Key Laboratory for Optical Materials and Photonic Devices,

Department of Chemistry, Capital Normal University,

Beijing 100048, P. R. China

Email: yswu@cnu.edu.cn

${ }^{5}$ Prof. Q. Peng, Prof. H. Dong, Dr. C. Gao

Beijing National Laboratory for Molecular Sciences

Institute of chemistry, Chinese Academy of Sciences

Beijing, 100049, P. R. China

Keywords: triplet energy gap, five-membered rings, ultrafast spectroscopy, singlet fission, hot exciton 


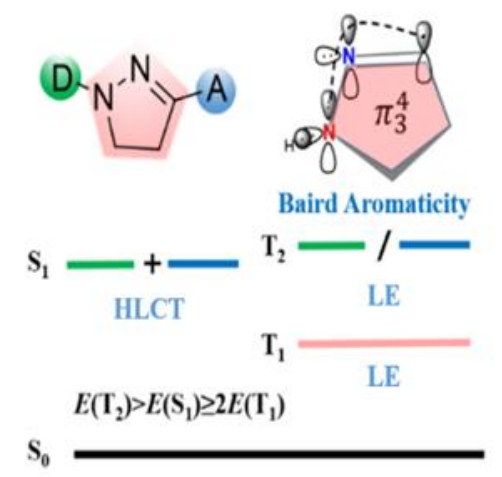

Table of Contents: We have proposed a facile strategy to achieve large triple gap by manipulating the aromaticity and the transition properties of the low-lying excited states, then theoretically designed and experimentally verified a series of donor-bridge-acceptor based on partially conjugated five-memebered rings with high aromaticity in $\mathrm{T}_{1}$ state to meet the harsh energy conditions of large triplet energy gap between $\mathrm{T}_{2}$ and $\mathrm{T}_{1}\left(\Delta E_{\mathrm{T}_{1} \mathrm{~T}_{2}}\right)$ for hot exciton materials and $E\left(\mathrm{~T}_{2}\right)>E\left(\mathrm{~S}_{1}\right) \geq 2 E\left(\mathrm{~T}_{1}\right)$ for singlet fission materials.

\begin{abstract}
Organic molecules with large gap between the excited singlet/triplet states can be applied in hot exciton emission and singlet fission to beat the spin statistics limit in optoelectronic devices. Herein, a novel strategy is proposed for achieving large triplet-triplet gap $\left(\Delta E\left(\mathrm{~T}_{1} \mathrm{~T}_{2}\right)\right)$ and singlet-triplet gap $\left(\Delta E\left(\mathrm{~S}_{1} \mathrm{~T}_{1}\right)\right)$ by manipulating the aromaticity of the low-lying excited states. Partially conjugated fivemembered heterocyclic rings are found to naturally have low $E\left(\mathrm{~T}_{1}\right)$ owing to high aromaticity obeying Baird's Rule. Utilizing such ring (pyrazoline) as a bridge and selecting various donor and acceptor moieties, numbers of derivatives have been theoretically designed with tunable emission colors, significantly large $\Delta E\left(\mathrm{~T}_{1} \mathrm{~T}_{2}\right)$ and $\Delta E\left(\mathrm{~S}_{1} \mathrm{~T}_{1}\right)$, and satisfying $E\left(\mathrm{~T}_{2}\right)>E\left(\mathrm{~S}_{1}\right) \geq 2 E\left(\mathrm{~T}_{1}\right)$. The ultrafast spectroscopy and sensitization measurements for one of them with blue fluorescence (named TPADBPrz) confirm the calculated prediction. This work opens a new avenue and provides molecular units to develop high-efficiency optoelectronic materials.
\end{abstract}

\title{
1. Introduction
}

Organic light-emitting diodes (OLEDs) have been successfully commercialized on displays and solidphase lighting due to their advantages. ${ }^{1-4}$ There has been a long-standing challenge to beat $25 \%$ statistical limit ${ }^{5}$ in the electroluminescence devices. Recently, nearly 100\% IQE has been obtained in the thermally-activated delayed fluorescence (TADF) materials for electrofluorescence. ${ }^{6-7}$ For TADF materials, efficient reverse intersystem crossing (RISC) is essential to convert triplet states to singlet manifold for fluorescence, which usually requires a significantly small singlet-triplet energy gap $\left(\Delta E_{\mathrm{ST}}\right){ }^{6}$ 
It is well known that such small $\Delta E_{\mathrm{ST}}$ can be generated by separating the highest occupied molecular orbital (HOMO) and lowest unoccupied molecular orbital (LUMO) in space, which concurrently leads to very weak oscillator strength, quite small emissive cross section and considerably long fluorescence lifetime. $^{6,8}$ The hybrid locally-excited charge transfer (HLCT) materials based on the hot exciton mechanism put forth by Ma and Yang are expected to make up for the deficiency, because more local excitation (LE) feature produces larger oscillator strength for fluorescence and RISC between higher excited singlet and triplet manifolds is more efficient without serious time delay. ${ }^{9}$ In this mechanism, the triplet excition is utilized through the RISC from higher triplet state to the singlet state. Thus, a large triplet-triplet gap $\Delta E\left(\mathrm{~T}_{1} \mathrm{~T}_{2}\right)$ is indispensable to efficiently suppress the nonradiative internal conversion from $\mathrm{T}_{2}$ to $\mathrm{T}_{1}$ which is a extremely harsh condition for organic compounds.

Very recently, the singlet fission (SF) materials are promising candidates for organic photovoltaics and electroluminescence because two low-energy triplet excited states $\left(\mathrm{T}_{1}\right)$ are generated from one highenergy singlet excited state $\left(S_{1}\right)$, overcoming the $34 \%$ Shockley-Queisser efficiency limit in solar cells ${ }^{10}$ and the $25 \%$ exciton utilization efficiency in OLED ${ }^{11}$. An efficient SF process basically requires the energy of $\mathrm{S}_{1}$ larger than twice that of $\mathrm{T}_{1}, E\left(\mathrm{~S}_{1}\right) \geq 2 E\left(\mathrm{~T}_{1}\right){ }^{12}$ At the same time, the energy condition of $E\left(\mathrm{~T}_{2}\right)>E\left(\mathrm{~S}_{1}\right)$ should also be met to avoid the intersystem crossing (ISC) from $\mathrm{S}_{1}$ to $\mathrm{T}_{2}$ and the recombination of $\mathrm{T}_{1}$ excitons. ${ }^{13}$ Overall, the HLCT and SF materials have similar energy requirements (see Figure 1), i.e., large $\Delta E\left(\mathrm{~T}_{1} \mathrm{~T}_{2}\right)$ and $\Delta E\left(\mathrm{~S}_{1} \mathrm{~T}_{1}\right)$. However, it is a formidable challenge to design molecules with large $\Delta E\left(\mathrm{~T}_{1} \mathrm{~T}_{2}\right)$ or $\Delta E\left(\mathrm{~S}_{1} \mathrm{~T}_{1}\right)$, let alone both.

In this work, we first demonstrate that some triplet-stabilized five-membered heterocyclic rings (i.e., pyrazoline) naturally have low $E\left(\mathrm{~T}_{1}\right)$ owing to high aromaticity in $\mathrm{T}_{1}$ obeying Baird's Rule ${ }^{14}$, and large energy gaps $\Delta E\left(\mathrm{~T}_{1} \mathrm{~T}_{2}\right)$ and $\Delta E\left(\mathrm{~S}_{1} \mathrm{~T}_{1}\right)$ due to the different transition properties among the $\mathrm{S}_{1}, \mathrm{~T}_{1}$ and $\mathrm{T}_{2}$ states. A series of donor-bridge-acceptor (D-B-A) systems are then designed based on pyrazoline ring as a bridge via introducing different donor or acceptor moieties (see Scheme 1). For the constructed pyrazoline derivatives, the low $E\left(\mathrm{~T}_{1}\right)$ is consistently maintained because the transition density of $\mathrm{T}_{1}$ is still localized in pyrazoline ring and the high $E\left(\mathrm{~T}_{2}\right)$ is generated via a transition over substituted donors or acceptors, which results in a large $\Delta E\left(\mathrm{~T}_{1} \mathrm{~T}_{2}\right)$. With that, a novel series of full-color emitters with large $\Delta E\left(\mathrm{~T}_{1} \mathrm{~T}_{2}\right)$ and $\Delta E\left(\mathrm{~S}_{1} \mathrm{~T}_{1}\right)$ are predicted through theoretical calculations and proved by transient absorption spectroscopy spectra of TPA-DBPrz, which, to the best of our knowledge, has not been previously reported. 

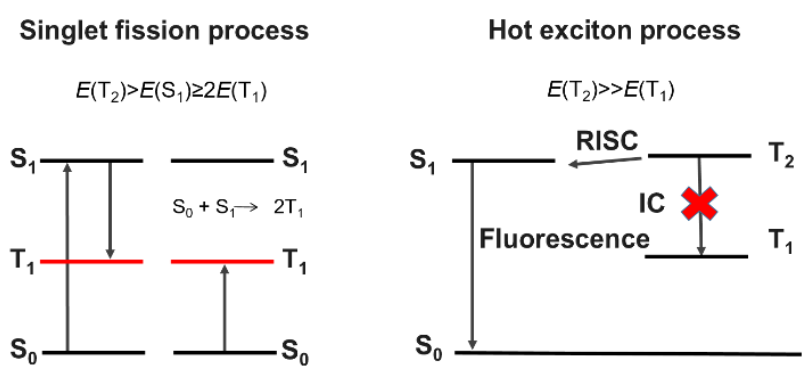

Figure 1. Schematic diagram of energy levels for the SF and HLCT materials.

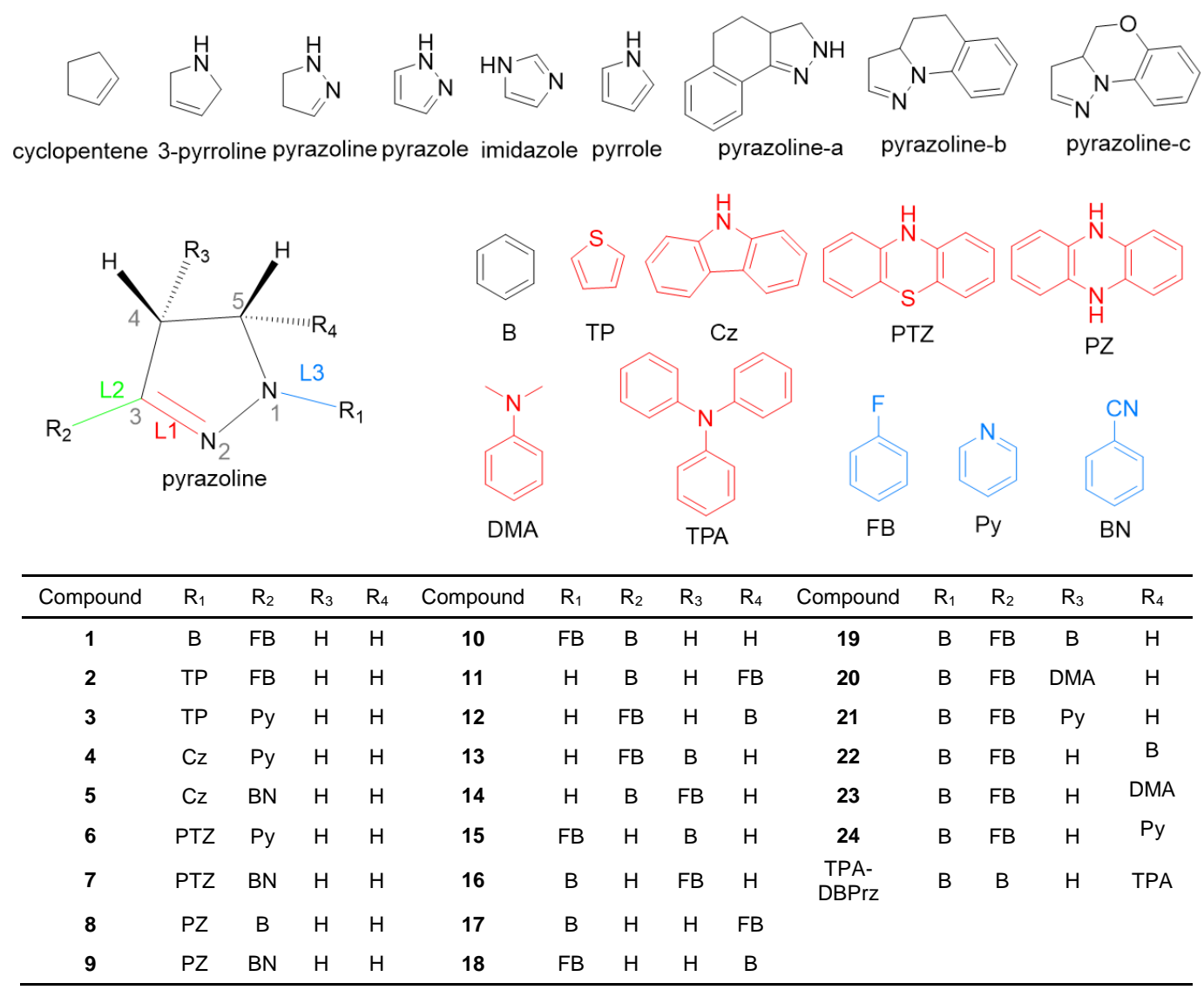

Scheme 1. Chemical structures of five-membered rings and constructed molecules in this work (red: donor; blue: acceptor).

\section{Results and Discussion}

\subsection{Five-membered heterocyclic rings with large triplet-triplet and singlet-tripelt gaps.}

The conjugated five-membered rings are frequently used as fundamental units in luminous molecules, such as cyclopentene, 3-pyrroline, pyrazoline, pyrazole, imidazole and pyrrole as shown in Scheme $\mathbf{1 .}$ The effects of molecular conjugation on the emissive spectra and efficiencies from $\mathrm{S}_{1}$ have been widely investigated in organic systems. ${ }^{15}$ However, the effects of molecular conjugation on the porperty and energy of triplet states have rarely been explored. We here systematically investigate the energy orders 
of low-lying excited triplet and singlet states of the five-membered rings with different degree of conjugation, including olefinic rings (cyclopentene), antiaromatic rings (3-pyrroline and pyrazoline) and aromatic rings (pyrazole, imidazole and pyrrole).

We optimize the geometrical structures via SA4-CASSCF/cc-PVTZ with active space $(10 \mathrm{e}, 10 \mathrm{o})$ and calculate the electronic structures via MS4-CASPT2/cc-PVTZ for the low-lying triplet and singlet states of these five-numbered rings, and the results are shown in Figure 2 and Table 1. It is seen in Figure 2a that cyclopentene, 3-pyrroline and pyrazoline are partially conjugated with transition densities mainly localized on double bond and lone-pair electrons, while pyrazole, imidazole and pyrrole are fully conjugated with transition density delocalized over the whole rings. Furthermore, it is found from Table 1 that the partially conjugated cyclopentene, 3-pyrroline and pyrazoline rings have low $E\left(\mathrm{~T}_{1}\right)$, which naturally meet the energy condition of $E\left(\mathrm{~T}_{1}\right)<<E\left(\mathrm{~T}_{2}\right)$ as required for HLCT materials and $2 E\left(\mathrm{~T}_{1}\right) \leq E\left(\mathrm{~S}_{1}\right)<E\left(\mathrm{~T}_{2}\right)$ as needed for SF materials; while the fully conjugated pyrazole, imidazole and pyrrole rings possess high $E\left(\mathrm{~T}_{1}\right)$ and relatively small $\Delta E\left(\mathrm{~T}_{1} \mathrm{~T}_{2}\right)$. Such energy orders are closely related to the natrue of the geometrial structures and electronic transition properies in the excited states. Upon excitation, the double bonds are elongated to a greater extent in cyclopentene $(0.130 \AA)$, 3-pyrroline $(0.158 \AA)$ and pyrazoline $(0.184 \AA$ ) than those (ca. $0.100 \AA$ ) in pyrazole, imidazole and pyrrole as shown in Figure $2 \mathrm{~b}$ and $\mathrm{S} 1$, which largely weaken the coupling between the two p-orbitals of the former, leading to low $E\left(\mathrm{~T}_{1}\right)$. These results are also consistent with Baird's Rule, in which the aromaticity is always changed upon triplet excitation and the aromatic triplet states are more stable with lower energy. ${ }^{14,16-17}$ Especailly, pyrazoline $\left(\pi_{3}^{4}\right)$ is a [4n] electron system, which is typically aromatic in $T_{1}$. The anisotropy of the induced current density ${ }^{18}$ (AICD) which can character the molecular aromaticity is calculated and plotted in Figure 2c. The clockwise ring currents in Figure 2c of cyclopentene, 3-pyrroline and pyrazoline rings indicate them to be aromatic while the anticlockwise ring currents in pyrazole, imidazole and pyrrole prove them to be antiaromatic in $\mathrm{T}_{1}$. Thus, according to Baird's Rule, cyclopentene, 3pyrroline and pyrazoline retain stabilized $\mathrm{T}_{1}$ states with low energy. Furthermore, the orbitals are energetically well-separated in these rings as shown in Figure 2d, especially, the energy gap between HOMO and HOMO-1 is very large. The $\mathrm{T}_{1}$ states are dominated by the transition from HOMO to LUMO while the $T_{2}$ states stem from the transition from deeper occupied orbitals to LUMO or higher unoccupied orbitals, which results in large $\Delta E\left(\mathrm{~T}_{1} \mathrm{~T}_{2}\right)$. All these results indicate cyclopentene, 3-pyrroline and pyrazoline are expected to be good triplet-stabilized candidates for ultra-large $\Delta E\left(\mathrm{~T}_{1} \mathrm{~T}_{2}\right)$. The similar conclusion can be obtained by the TDA-DFT approach as shown in supporting information (SI). 
(a)

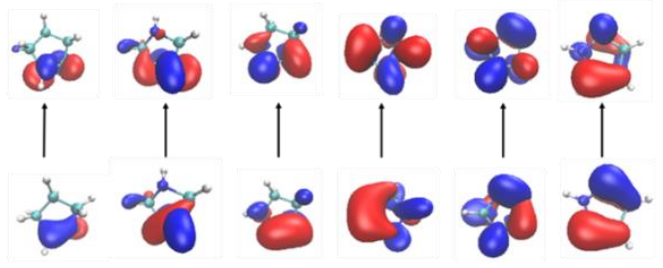

(b)

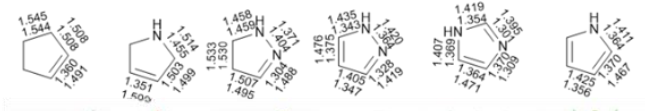

(c)

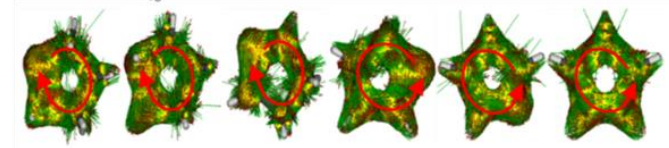

(d)

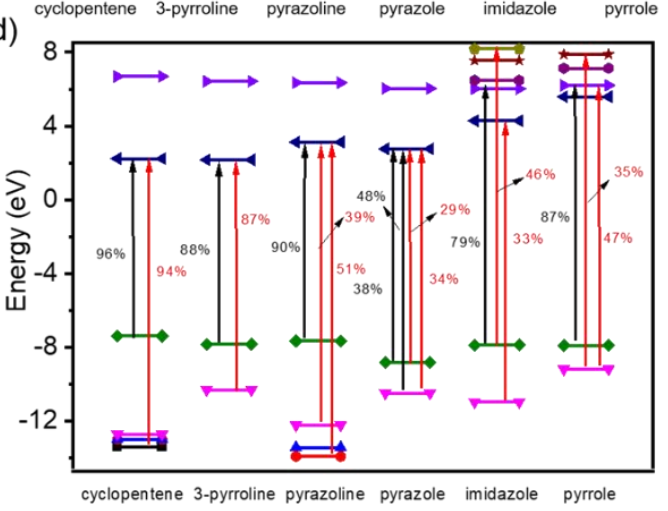

Figure 2. a) Nature transition orbitals (NTOs) of $\mathrm{T}_{1}$ at $\mathrm{T}_{1}$-geometry; b) Optimized bond lengths (Inside: S0-geometry; Outside: $\mathrm{T}_{1}$-geometry); c) AICD plots of six five-numbered rings in $\mathrm{T}_{1}$ state in which the induced current density vectors denoted by the arrows; and d) frontier orbitals energies and transitions of $\mathrm{T}_{1}$ (black) and $\mathrm{T}_{2}$ (red) states.

Table 1. Vertical excitation energies (Unit: $\mathrm{eV}$ ) of six five-numbered rings at $\mathrm{T}_{1}$-geometry.

\begin{tabular}{cccccc}
\hline Compound & $E\left(\mathrm{~T}_{1}\right)$ & $E\left(\mathrm{~T}_{2}\right)$ & $E\left(\mathrm{~S}_{1}\right)$ & $2 E\left(\mathrm{~T}_{1}\right)$ & $\Delta E\left(\mathrm{~T}_{1} \mathrm{~T}_{2}\right)$ \\
\hline cyclopentene & 1.01 & 6.04 & 3.77 & 2.02 & 5.03 \\
3-pyrroline & 1.26 & 4.09 & 3.56 & 2.52 & 2.83 \\
pyrazoline & 1.54 & 4.68 & 3.55 & 3.08 & 3.14 \\
pyrazole & 2.84 & 4.43 & 4.97 & 5.68 & 1.59 \\
imidazole & 3.44 & 5.10 & 5.44 & 6.88 & 1.66 \\
pyrrole & 3.35 & 4.84 & 5.34 & 6.70 & 1.49 \\
\hline
\end{tabular}

We further calculate the low-lying excited states of the extended systerms (pyrazoline-a, pyrazoline-b and pyrazoline-c in Scheme 1) based on pyrazoline ring at TDA/LC- $\omega P B P^{*} / 6-31 G(d)$ level. They all exhibit much lower energy of $T_{1}$ than those of $S_{1}$ and $T_{2}$ (Table S3) because the $T_{1}$ mainly originates from pyrazoline ring (Figure $\mathrm{S} 3 \mathrm{a}$ ), while $\mathrm{T}_{2}$ and $\mathrm{S}_{1}$ are significantly changed by the introduced chemical groups. These results further demonstrate the partly conjugated rings can serve as triplet-stabilized moieties for the HLCT and SF materials.

\subsection{Construction of pyrazoline derivatives with large triplet-triplet and singlet-triplet gaps.}


Pyrazoline derivatives have been investigated as hole-transport materials ${ }^{19}$, fluorescent sensor $^{20}$ and medicine $^{21}$ owing to high stability and wonderful photophysical properties. Very few cyclopentene and 3-pyrroline derivatives have been reported in the literature, let alone their applications in the materials science. Hence, in this work, pyrazoline ring is used as a bridge (B) to construct 25 compounds by introducing different donor (D) and acceptor (A) groups, namely, D-B-A type compounds (see Scheme 1). Among them, the electron-withdrawing ability of the acceptors becomes stronger from $\mathrm{FB}$, Py to $\mathrm{BN}$ owing to lower LUMO energies, and the electron-donating ability of the donors becomes stronger from TP, Cz, PTZ to PZ due to higher HOMO energies (Table S4).

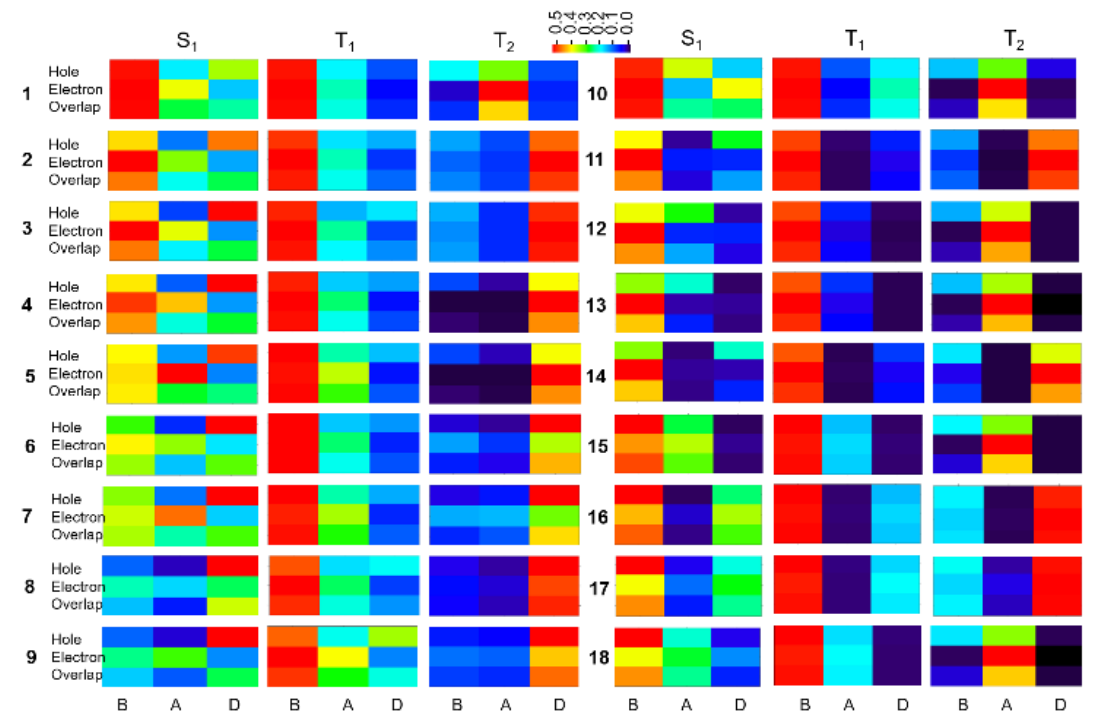

Figure 3. Hole-electron distribution heat maps of 1-18.

Compounds 1-9 with different D or A at the same substituted 1- and 3-positions all possess with nearly coplanar conformations with high rigidity. Upon excitation, significant modifications occur on three bond lengths rather than angles and dihedral angles, as seen in Table S5. These three bond lengths are $\mathrm{C}=\mathrm{N}$ in pyrazoline ring $\left(\mathrm{L}_{1}\right)$, the bond linking $B$ and $A\left(L_{2}\right)$ and the bond linking $B$ and $D\left(L_{3}\right)$, respectively, as illustrated in Scheme 1. From $S_{0}$-geometry to $T_{1}$-geometry, $L_{1}$ is considerably elongated by 0.081-0.136 $\AA$, which is similar to the change of single pyrazoline ring, while $\mathrm{L}_{2}$ and $\mathrm{L}_{3}$ are slightly shortened by 0.002-0.07 $\AA$ as seen in Table S5. The hole-electron analyses are carried out to unravel the transition nature of the low-lying excited states through hole-electron distribution heat maps ${ }^{22}$ and natural transition orbitals (NTOs) as plotted in Figure 3 and Figure S4, respectively. It is seen from two figures that for all the molecules $T_{1}$ is a locally excited (LE) state originating from pyrazoline ring, which results in their $E\left(\mathrm{~T}_{1}\right)$ very close to that of single pyrazoline ring, namely, ca. $1.50 \mathrm{eV}$, as shown in Figure $4 \mathrm{a}$. $\mathrm{T}_{2}$ is a $\mathrm{LE}$ state mainly stemming from either acceptor or donor group, which has much wider energy-gap than that of pyrazoline ring. Thus, $E\left(\mathrm{~T}_{1}\right)$ is much smaller than $E\left(\mathrm{~T}_{2}\right)$. By contrast, $\mathrm{S}_{1}$ acquires a delocalized transition with partial charge-transfer $(\mathrm{CT})$ character. The resultant $E\left(\mathrm{~S}_{1}\right)$ is smaller than $E\left(\mathrm{~T}_{2}\right)$ as seen in 
Figure 4a. Furthermore, the difference in the nature of electronic configuration between $T_{2}$ and $S_{1}$ states can give rise to large spin-orbit coupling (SOC), which facilitates the mutual conversion between them. Overall, the nine compounds all meet the energy conditions of $E\left(\mathrm{~T}_{1}\right)<<E\left(\mathrm{~T}_{2}\right)$ and $2 E\left(\mathrm{~T}_{1}\right) \leq E\left(\mathrm{~S}_{1}\right)<E\left(\mathrm{~T}_{2}\right)$, indicating them to be good candidates for HLCT or SF molecules. In addition, while retaining large $\Delta E\left(\mathrm{~T}_{1} \mathrm{~T}_{2}\right)$ and $\Delta E\left(\mathrm{~S}_{1} \mathrm{~T}_{1}\right)$, the emissive wavelengths vary in a wide visible region of $2.30 \sim 3.27 \mathrm{eV}$ (Table S6), which enriches the HLCT or SF systems with multicolor emission light.

Among the nine compounds, compound 1 has the largest $\Delta E\left(\mathrm{~T}_{1} \mathrm{~T}_{2}\right)(2.19 \mathrm{eV})$ with the substituents $\mathrm{BF}$ and $\mathrm{B}$. Hence, we change the substituted position of BF and B (compounds 10-18 in Scheme 1) to investigate the position dependence of the substituents on the properties of the low-lying excited states in Figure 4b. It can be seen that (i) the energy relationship of $E\left(\mathrm{~T}_{1}\right)<<E\left(\mathrm{~T}_{2}\right)$ is still maintained with $\Delta E\left(\mathrm{~T}_{1} \mathrm{~T}_{2}\right)$ larger than $1.91 \mathrm{eV}$, which is independent on the positions of $\mathrm{BF}$ and $\mathrm{B}$ in the pyrazoline ring; (ii) the $2 E\left(\mathrm{~T}_{1}\right) \leq E\left(\mathrm{~S}_{1}\right)<E\left(\mathrm{~T}_{2}\right)$ is well held except for 11 and 14 with $\mathrm{B}$ at the 3- site and BF at the 4- or 5- sites of pyrazoline ring; (iii) relative to compound 1 and 10, the $E\left(\mathrm{~S}_{1}\right)$ and $E\left(\mathrm{~T}_{2}\right)$ are raised while the $E\left(\mathrm{~T}_{1}\right)$ fluctuates between 1.30 and $1.71 \mathrm{eV}$ for compounds 11-18. Thus, it can be predicted that among these systems, compounds 12, 15 and 18 would be excellent HLCT materials because of large $\Delta E\left(\mathrm{~T}_{1} \mathrm{~T}_{2}\right)$ that blocks the internal conversion from $\mathrm{T}_{2}$ to $\mathrm{T}_{1}$ and small $\Delta E\left(\mathrm{~S}_{1} \mathrm{~T}_{2}\right)$ that facilitates the intersystem crossing from $T_{2}$ to $S_{1}$; and Compounds 10, 16 and 17 would act as excellent SF materials owing to not only $2 E\left(\mathrm{~T}_{1}\right) \leq E\left(\mathrm{~S}_{1}\right)$ but also large $\Delta E\left(\mathrm{~S}_{1} \mathrm{~T}_{2}\right)$. The reason of large $\Delta E\left(\mathrm{~T}_{1} \mathrm{~T}_{2}\right)$ in 10-18 is the same with that in 19 (Figure 3 and Figure S4).

The effect of the number of substituents on the excited-state property are further studied through adding donor or acceptor at 4- or 5-site of pyrazoline ring when B and BF are fixed at 1- and 3-site, respectively (compounds 19-24 in Scheme 1), and the resultant excitation energies and NTOs are given in Table S6 and Figures $4 \mathrm{c}, \mathrm{S} 4$. The $E\left(\mathrm{~S}_{1}\right), E\left(\mathrm{~T}_{1}\right), E\left(\mathrm{~T}_{2}\right)$ of these compounds 19-24 are all similar to those of compound $\mathbf{1}$, which suggests the substituents linked to the $\mathrm{sp}^{3}$ hybridized carbon atom of the pyrazoline $\left(\mathrm{R}_{3}\right.$ and $\mathrm{R}_{4}$ ) have no effect on the excited-state properties while the ones at the conjugated part of the pyrazoline ring play important roles. Therefore, it is declared that the low-lying excited-state properties can be tuned by changing $\mathrm{R}_{1}$ and $\mathrm{R}_{2}$ at 1 - and 3- sites of pyrazoline ring, and other physical properties such as crystallization ability, glass-transition temperature etc. can be controlled via adding $\mathrm{R}_{3}$ or $\mathrm{R}_{4}$ at 4and 5-sites. Thus, excellent HLCT or SF materials can be properly designed for optoelectronic devices. 

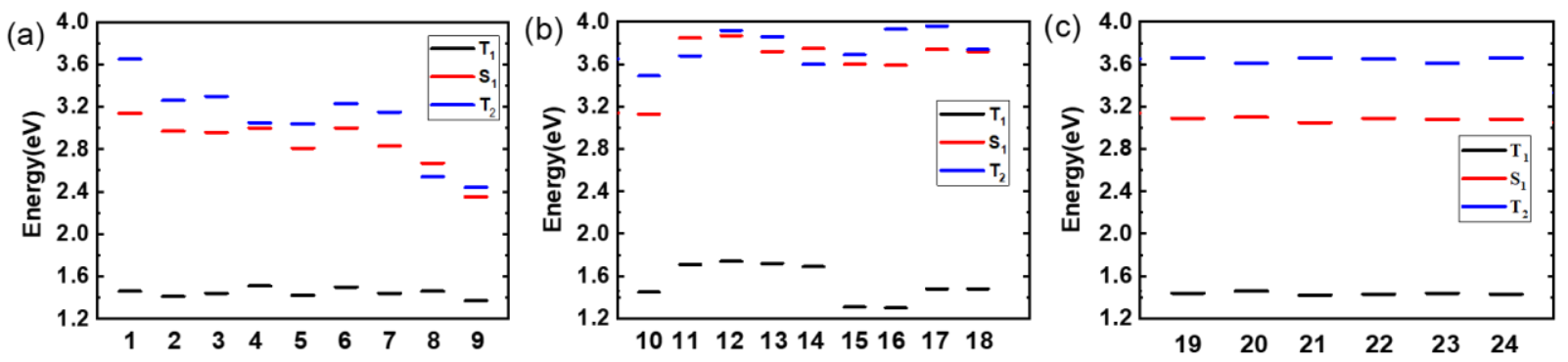

Figure 4. Excitation energies of the compounds at T1-geometry at TDA/LC- $\omega$ PBE*/6-31G(d) level.

\subsection{Experimental validation of theoretically designed TPA-DBPrz molecule.}

Keeping the above design strategy in mind, we build a compound named as TPA-DBPrz with $\mathrm{R}_{1}=\mathrm{B}$, $\mathrm{R}_{2}=\mathrm{B}, \mathrm{R}_{3}=\mathrm{H}$ and $\mathrm{R}_{4}=\mathrm{TPA}$, which has properly large molecular weight to meet the requirement for the application in optoelectronic devices. The low-lying excited states are calculated at MS4CASPT2(8,8)/cc-PVDZ level and the results are given in Figure 5a, including energy levels, transition properties and involved frontier orbitals. The $S_{1}$ state has a hybrid transition character with LE on pyrazoline and CT from 1-site phenyl to 3 -site phenyl ring. The $\mathrm{T}_{1}$ state stems from LE on pyrazoline ring, as well as a marginal contribution from two phenyl rings. The $\mathrm{T}_{2}$ state comes from the electron transition on TPA moiety. The resultant $E\left(\mathrm{~S}_{1}\right), E\left(\mathrm{~T}_{2}\right)$ and $E\left(\mathrm{~T}_{1}\right)$ are $2.68,1.55$, and $3.23 \mathrm{eV}$ based on $\mathrm{T}_{1-}$ goemetry, respectively, leading to large $\Delta E\left(\mathrm{~T}_{1} \mathrm{~T}_{2}\right)$ of $1.68 \mathrm{eV}$. In addition, the oscillator strength $(f)$ of $\mathrm{T}_{1} \rightarrow \mathrm{T}_{2}$ transiton is a significant value $(f=0.0007)$, which indicates the effective absorption from $\mathrm{T}_{1}$ to $\mathrm{T}_{2}$. To prove our theoretical predictions, we synthesized TPA-DBPrz compound and measured its photophysical properties. The synthetic route of TPA-DBPrz is shown in Scheme S1 and it is unambiguously characterized by ${ }^{1} \mathrm{H} \mathrm{NMR},{ }^{13} \mathrm{C}$ NMR, high resolution mass spectrometry and elemental analysis as given in SI. The ultraviolet-visible absorption and photoluminescence (PL) spectra of TPADBPrz in chloromethane (DCM) solution are plotted in Figure 5b. The PL shows a blue emission with a emission maximum at $453 \mathrm{~nm}$, very close to the theoretical value. Femtosecond transient absorption spectroscopy (fs-TA) of TPA-DBPrz in dichloromethane (DCM) is performed upon excitation at $400 \mathrm{~nm}$ and it is found that there appear two types of features: short-lived excited state absorption (ESA) band at about $582 \mathrm{~nm}$, and two gradually increasing ESA bands at both $527 \mathrm{~nm}$ and $830 \mathrm{~nm}$ (see Figure 5d-e, S5). The long-lived component dominates the spectrum after $250 \mathrm{ps}$ and lasting for several ns. It is seen that (i) the excited state corresponding to $582 \mathrm{~nm}$ firstly generates upon excitation and then disappear rapidly, which can be attributed to the transition of $S_{1} \rightarrow S_{n}$; (ii) the transient species corresponding to 527 $\mathrm{nm}$ and $830 \mathrm{~nm}$ is populated from the $\mathrm{S}_{1}$ state, which can be hypothetically assigned to the transition from $T_{1}$ to $T_{n}$ state. In order to confirm this assignment, we further obtain the sensitized $T_{1}$ state of the TPA- 
DBPrz using a phosphorescence dye $\mathrm{Ru}(\mathrm{bpy}){ }_{3} \mathrm{Cl}_{2}$ with a $E\left(\mathrm{~T}_{1}\right)(2.1 \mathrm{eV}) .{ }^{23}$ (detailed in Figure S7). It is shown that the sole $\mathrm{Ru}(\mathrm{bpy})_{3} \mathrm{Cl}_{2}$ show no ESA band at $527 \mathrm{~nm}$ and $830 \mathrm{~nm}$ while $\mathrm{Ru}(\mathrm{bpy})_{3} \mathrm{Cl}_{2} \& \mathrm{TPA}-$ DBPrz exhibit a long-lived absorption band (Figure S6) at 830nm (1.49eV) and a strong and long-lived ESA band (Figure 5c) at 527nm, corresponding to the lowest excited state of TPA-DBPrz $\left(\mathrm{T}_{1}\right)$ sensitized by $\mathrm{Ru}(\text { bpy })_{3} \mathrm{Cl}_{2}$. This is coincident with the calculated energies of the $\mathrm{T}_{1} \rightarrow \mathrm{T}_{2}(1.68 \mathrm{eV})$ and $\mathrm{T}_{1} \rightarrow \mathrm{T}_{\mathrm{n}}$ transitions of TPA-DBPrz mentioned above. The rate constant for intersystem crossing from $\mathrm{S}_{1}$ to $\mathrm{T}_{1}$ can be determined from the rise evolution of $830-\mathrm{nm}$ curve, yielding the $k_{\text {ISC }}$ of $103 \mathrm{ps}^{-1}$. These results confirm that the theoretically designed TPA-DBPrz possesses large $\Delta E\left(\mathrm{~T}_{1} \mathrm{~T}_{2}\right)$, potentially important for HLCT and SF application.

(a)

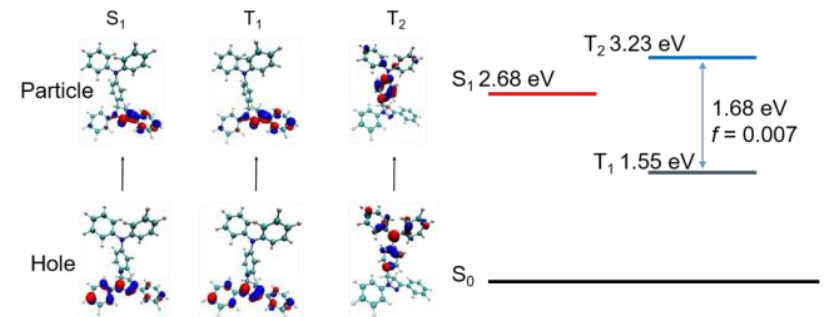

(b)

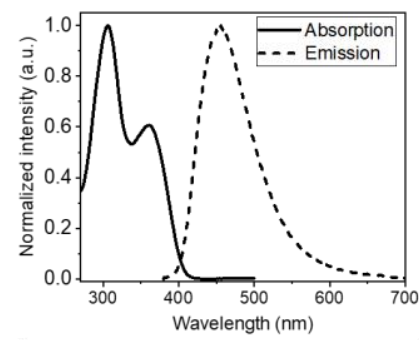

(c)

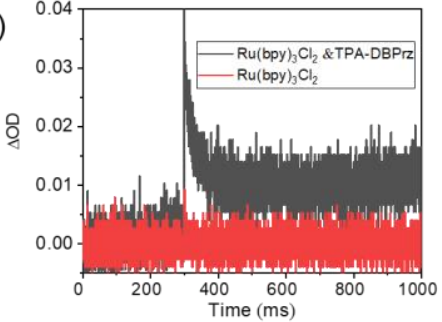

(d)

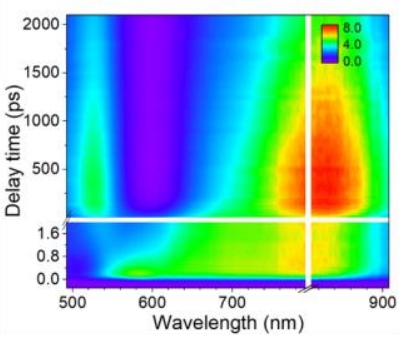

(e)

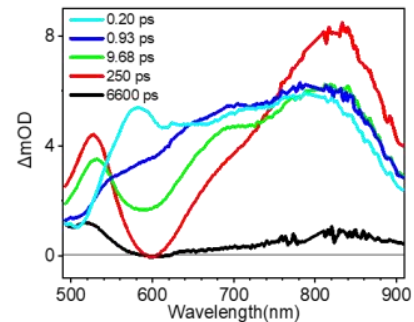

Figure 5. a) Nature transition oribtals (NTOs), energy levels and the oscillator strength $(f)$ of TPA-DBPrz based on $\mathrm{T}_{1}$-goemetry. b) Normalized UV-vis and PL spectra in DCM solution; c) ns-TA measurement of $\mathrm{Ru}(\mathrm{bpy})_{3} \mathrm{Cl}_{2}(20 \mathrm{uM})$ and $\mathrm{Ru}(\mathrm{bpy})_{3} \mathrm{Cl}_{2}(20 \mathrm{uM})$ \&TPA-DBPrz $(1 \mathrm{mM})$ in DCM monitored at $527 \mathrm{~nm}$. d) and e) fs-TA spectra and corresponding dynamics curves of TPA-DBPrz in the DCM solution (10 $\mu \mathrm{M})$.

\section{Conclusion}

To conclude, we propose an excited-state aromaticity strategy to design the compounds with large triplettriplet and singlet-triplet gaps which are of potential application in SF and hot exciton materials. We first investigate the energy orders in the low-lying excited states of a seires of typical five-membered rings 
with different degree of conjugation using the state-of-the-art quantum chemistry approaches. It is found that the partly conjugated five-membered heterocyclic rings are expected to be good triplet-stabilized candidates because of elongated double bond (by 0.130 0.184 Angstrom) and high aromaticity in $\mathrm{T}_{1}$. Then, we choose pyrazoline ring as a bridge and design 25 molecules of donor-bridge-acceptor (D-B-A) type through substituting donors and acceptor at different site positions. The calculated results indicate that $\mathrm{T}_{1}$ is a locally excited state originating from pyrazoline ring, which maintains low $E\left(\mathrm{~T}_{1}\right)$ (very close to that of single pyrazoline ring, ca. $1.50 \mathrm{eV}$ ), $\mathrm{T}_{2}$ is a locally excited state mainly stemming from either acceptor or donor moiety, which generates high $E\left(\mathrm{~T}_{2}\right)$, while $\mathrm{S}_{1}$ is a hybrid excited state with local excitation and charge transfer character. Consequently, the $E\left(\mathrm{~T}_{2}\right)$ is much higher than $\mathrm{T}_{1}$ and the $E\left(\mathrm{~S}_{1}\right)$ is slightly smaller than $E\left(\mathrm{~T}_{2}\right)$, which result in large $\Delta E\left(\mathrm{~T}_{1} \mathrm{~T}_{2}\right)$, and $\Delta E\left(\mathrm{~S}_{1} \mathrm{~T}_{1}\right)$ and meeting $2 E\left(\mathrm{~T}_{1}\right) \leq E\left(\mathrm{~S}_{1}\right)<E\left(\mathrm{~T}_{2}\right)$. Finally, we synthesize a designed TPA-DBPrz molecule and investigate its photophysical properties by theoretical calculations and experimental measurements. The fs-TA spectra of TPA-DBPrz in DCM and the ns-TA spectra of TPA-DBPrz\&Ru(bpy $)_{3} \mathrm{Cl}_{2}$ in DCM both show the absorption from $\mathrm{T}_{1}$ to $\mathrm{T}_{2}$ at $830 \mathrm{~nm}(1.49 \mathrm{eV})$, which fully proves the theoretically predicted $\Delta E\left(\mathrm{~T}_{1} \mathrm{~T}_{2}\right)$ of $1.68 \mathrm{eV}$.

In summary, using reliable quantum chemistry methods, we have successfully identified five-membered heterocyclic rings which can naturally stabilize triplet state, based on which we have designed 25 compounds that all exhibit large $\Delta E\left(\mathrm{~T}_{1} \mathrm{~T}_{2}\right)$ and $\Delta E\left(\mathrm{~S}_{1} \mathrm{~T}_{1}\right)$ and thus serve as promising candidates for hot exciton and SF materials. This work will provide a new route and a number of novel molecular cores for designing high-performance hot exciton and SF materials in practice.

\section{Acknowledgements}

This work was supported by the National Natural Science Foundation of China, Grant Nos. 21788102, 21973099 and 22003030, as well as by the Ministry of Science and Technology of China through the National Key R\&D Plan, Grant No. 2017YFA0204501 and 2020YFB0204802. QO is also funded by China Postdoctoral Science Foundation Grant No. 2020M670280.

\section{Author contributions}

ZS, QP and QS conceived the project. QS designed molecules and carried out the calculations. QO, DH, and LW helped with the calculation process and provided inspiring suggestions for improvement. YC and YW synthesized the molecule. CG, YW and LH conducted the ultrafast spectroscopy and sensitization measurements. All authors contributed to data analysis and write the paper.

\section{Competing interests}

The authors declare no competing interests.

\section{References}


1. Tang, C. W.; VanSlyke, S. A., Organic electroluminescent diodes. Appl. Phys. Lett. 1987, 51 (12), 913-915.

2. $\quad$ Reineke, S.; Lindner, F.; Schwartz, G.; Seidler, N.; Walzer, K.; Lussem, B.; Leo, K., White organic light-emitting diodes with fluorescent tube efficiency. Nature 2009, 459, 234-238.

3. Sun, Y.; Giebink, N. C.; Kanno, H.; Ma, B.; Thompson, M. E.; Forrest, S. R., Management of singlet and triplet excitons for efficient white organic light-emitting devices. Nature 2006, 440, 908-912. 4. $\quad$ Evans, R. C.; Douglas, P.; Winscom, C. J., Coordination complexes exhibiting room-temperature phosphorescence: Evaluation of their suitability as triplet emitters in organic light emitting diodes. Coordin. Chem. Rev. 2006, 250, 2093-2126.

5. $\quad$ Shuai, Z., Beljonne, D., Silbey, R. J., and Brédas, J. L., Singlet and Triplet Exciton Formation Rates in Conjugated Polymer Light-Emitting Diodes. Phys. Rev. Lett. 2000, 84, 131-134.

6. Uoyama, H.; Goushi, K.; Shizu, K.; Nomura, H.; Adachi, C., Highly efficient organic lightemitting diodes from delayed fluorescence. Nature 2012, 492, 234-238.

7. Endo, A.; Ogasawara, M.; Takahashi, A.; Yokoyama, D.; Kato, Y.; Adachi, C., Thermally activated delayed fluorescence from $\mathrm{Sn}(4+)$-porphyrin complexes and their application to organic light emitting diodes--a novel mechanism for electroluminescence. Adv. Mater. 2009, 21, 4802-486.

8. Kaji, H.; Suzuki, H.; Fukushima, T.; Shizu, K.; Suzuki, K.; Kubo, S.; Komino, T.; Oiwa, H.; Suzuki, F.; Wakamiya, A.; Murata, Y.; Adachi, C., Purely organic electroluminescent material realizing $100 \%$ conversion from electricity to light. Nat. Commun. 2015, 6, 8476.

9. Pan, Y.; Li, W.; Zhang, S.; Yao, L.; Gu, C.; Xu, H.; Yang, B.; Ma, Y., High Yields of Singlet Excitons in Organic Electroluminescence through Two Paths of Cold and Hot Excitons. Adv. Opt. Mater. 2014, 2, 510-515.

10. Congreve, D. N.; Lee, J.; Thompson, N. J.; Hontz, E.; Yost, S. R.; Reusswig, P. D.; Bahlke, M. E.; Reineke, S.; Van Voorhis, T.; Baldo, M. A., External quantum efficiency above $100 \%$ in a singletexciton-fission-based organic photovoltaic cell. Science 2013, 340, 334-337.

11. Nagata, R.; Nakanotani, H.; Potscavage, W. J., Jr.; Adachi, C., Exploiting Singlet Fission in Organic Light-Emitting Diodes. Adv. Mater. 2018, e1801484.

12. Millicent B. Smith , J. M., Singlet Fission. Chem. Rev. 2010, 110, 6891-6936.

13. Akdag, A.; Havlas, Z.; Michl, J., Search for a small chromophore with efficient singlet fission: biradicaloid heterocycles. J. Am. Chem. Soc. 2012, 134, 14624-14631.

14. Baird, N. C., Quantum Organic Photochemistry. II. Resonance and Aromaticity in the Lowest $3 \pi \pi^{*}$ State of Cyclic Hydrocarbons. J. Am. Chem. Soc. 1972, 94, 4941-4948.

15. Xie, Y.; Zhang, T.; Li, Z.; Peng, Q.; Yi, Y.; Shuai, Z., Influences of Conjugation Extent on the Aggregation-Induced Emission Quantum Efficiency in Silole Derivatives: A Computational Study. Chem. Asian. J. 2015, 10, 2154-61.

16. Ottosson, H., Organic photochemistry: Exciting excited-state aromaticity. Nat. Chem. 2012, 4, 969-971.

17. Fallon, K. J.; Budden, P.; Salvadori, E.; Ganose, A. M.; Savory, C. N.; Eyre, L.; Dowland, S.; Ai, Q.; Goodlett, S.; Risko, C.; Scanlon, D. O.; Kay, C. W. M.; Rao, A.; Friend, R. H.; Musser, A. J.; Bronstein, H., Exploiting Excited-State Aromaticity To Design Highly Stable Singlet Fission Materials. J. Am. Chem. Soc. 2019, 141, 13867-13876.

18. Daniel Geuenich, K. H., Felix Kohler, and Rainer Herges, Anisotropy of the Induced Current Density (ACID), a General Method To Quantify and Visualize Electronic Delocalization. Chem. Rev. 2005, 105, 3758-3772.

19. Cherpak, V.; Stakhira, P.; Khomyak, S.; Volynyuk, D.; Hotra, Z.; Voznyak, L.; Dovbeshko, G.; Fesenko, O.; Sorokin, V.; Rybalochka, A.; Oliynyk, O., Properties of 2,6-di-tert.-butyl-4-(2,5-diphenyl3,4-dihydro-2H-pyrazol-3-yl)-phenol as hole-transport material for life extension of organic light emitting diodes. Opt. Mater. 2011,33, 1727-1731. 
20. Fahrni, C. J.; Yang, L.; VanDerveer, D. G., Tuning the photoinduced electron-transfer thermodynamics in 1,3,5-triaryl-2-pyrazoline fluorophores: X-ray structures, photophysical characterization, computational analysis, and in vivo evaluation. J. Am. Chem. Soc. 2003, 125, 3799-3812. 21. Zhu, S. L.; Wu, Y.; Liu, C. J.; Wei, C. Y.; Tao, J. C.; Liu, H. M., Design and stereoselective synthesis of novel isosteviol-fused pyrazolines and pyrazoles as potential anticancer agents. Eur. J. Med. Chem. 2013, 65, 70-82.

22. Liu, Z.; Lu, T.; Chen, Q., An sp-hybridized all-carboatomic ring, cyclo[18]carbon: Electronic structure, electronic spectrum, and optical nonlinearity. Carbon 2020, 165, 461-467.

23. Xu, K.; Zhao, J.; Moore, E. G., Photo-induced electron transfer in a diamino-substituted $\mathrm{Ru}(\mathrm{bpy}) 3[\mathrm{PF} 6] 2$ complex and its application as a triplet photosensitizer for nitric oxide (NO)-activated triplet-triplet annihilation upconversion. Photochem. Photobiol. Sci. 2016, 15, 995-1005. 